\title{
Comparative Study of Diverse CFO Estimation Techniques in OFDM System
}

\author{
Aanchal Jhingan, Lavish Kansal and Navdeep Singh Dhaliwal* \\ School of Electronics and Electrical Engineering, Lovely Professional University, Phagwara - 144411, \\ Punjab, India; aanchal.20186@lpu.co.in, lavish.15911@lpu.co.in,9avdeep.dhaliwal@gmail.com
}

\begin{abstract}
A multiplexing technique named Orthogonal Frequency Division Multiplexing (OFDM) has been widely used in a broadband wireless communication system. This technique can provide the larger data rates with the capability of showing enough strength to the impairments caused by the radio based channels. The OFDM based systems are supposed to be sensitive towards the errors that originate due to the frequency mismatch in the form of the Carrier Frequency Offset (CFO) effect. That further leads to an effect named Inter-Carrier Interference (ICI). ICI mainly occurs due to the inconsistency in the frequencies of the local oscillators that are present together at the transmitter and the receiver side. For compensating the effect of the CFO, the researchers have proposed different types of methods and algorithms used for estimation and its correction by now. In this research, the reason for the generation of the CFO and its effects are shown on the performance of the system is studied. The main algorithms and techniques for the CFO estimation and correction are reviewed and discussed in the literature briefly and also discussed a proposed and an efficient method for the estimation and compensation of the $\mathrm{CFO}$ is proposed.
\end{abstract}

Keywords: BER, CFO, ICI, ISI, OFDM

\section{Introduction}

OFDM is one of the modulation techniques that use multi- carriers in its modulation which makes it a widely used technique in the wireless systems. In its modulation process, the carriers in the signal are supposed to be orthogonal in respective to each other, and that result in the increase of the efficiency of the wireless system in terms of bandwidth. The frequency channel during the transmission is converted into a group of narrow band channels (Flat Fading), dividing one channel across each of the subchannel. The modulation and the demodulation process is done by the Inverse Discrete Fourier Transform (IDFT) and Discrete Fourier Transform (DFT) respectively 2.2 . The FFT techniques employed makes it computationally efficient. The guard intervals insertion in OFDM systems leads to the improvement in inter-symbol/channel interference. Further, the symbol period extension of made easier the code synchronization in the system. CFO is one of the drawbacks in OFDM, which limits its performance and bandwidth efficiency which needs to be mitigated for the better OFDM system performance. OFDM is mainly a technique that is used to achieve the high data rates over the multi-carrier based communication by transmitting a sum of overlapped sub-carriers which are orthogonal to each other. The orthogonal between the carriers helps in improving the spectral efficiency of the carriers. As long as the carriers are assumed to be orthogonal to each other, it is easier for the receiver to recover the data from each of the sub-carrier. So that is the main reason due to which the Orthogonality between the carriers needs to be maintained in the system.

In the next step of this scheme, the transmission of the sum of the carriers which are orthogonal and are overlapped to each other is done in a parallel manner in the narrow band pattern. Further, each of the carriers is taken for the modulation process by using the Quadrature Amplitude or Phase-Shift Keying Modulation technique

*Author for correspondence 
at a very low symbol rate considering the rate which is necessary for the complete data stream with the condition of maintaining the data rates in the same bandwidth in a similar way to the single carrier modulation schemes. It is an efficient computational method used to perform modulation and demodulation functions by using FFT techniques. An addition of guard period in the symbol helps in mitigating the inter-symbol/channel interferences in the symbol. Further, the extension of the symbol is done relating to the sum of the carriers by making code synchronization in the carriers. The data stream of the higher rate is sliced up in some lower data rate streams and these streams all together are taken for transmission over a parallel medium. A reduction in the sum of dispersion in the time occurs due to the delay in the multipath that increases the symbol duration for the sub-carriers. The whole bandwidth which is available for the transmission of carriers is divided equally among the sub-carriers. As the modulation of the carriers is done at the lower rates and the separation between them gives a compact and an efficient spectra utilization of the bandwidth which is available.

In the recent years, the OFDM technique has become very popular as an efficient transmission technique due to its qualities like the highly efficient in the spectra's, the robustness in against to the fading that occurs over the multipath and the noises (impulsive) that makes it more useful method for the high rate data based systems. There are some factors like the CFO and the noise in the phase makes OFDM technique very much sensitive to the frequency offset based factors that make it loose the Orthogonality factors among the subcarriers. The Orthogonality maintenance is done by interpreting the signal in the OFDM by using the Fourier transform procedures i.e. the IDFT and DFT. In the OFDM based systems, a huge number of closely spaced and placed in a narrow band carriers are taken for the transmission process, provided an assurance of no cross talk from other subchannels as the carriers are placed at the frequency which is centrally defined at each of the sub-channels. First of all, at the transmission side, the single input bit stream is taken for the multiplexing scheme which divides it into a number of the symbol streams which are represented by the symbol $\mathrm{N}$, with each one of the carriers has some specific symbol period which is further represented by the factor ${ }^{\prime} T_{s}^{\prime}$. Then each of the multiplexed symbols is taken further for the modulation process which makes it synchronized and a serial to the parallel conversion is done. The carriers spacing is defined in an interval of [0, $T_{S}$ ] in the form of frequency as $1 / \mathrm{N}^{T_{S}}$ and this spacing defined in this particular interval makes the carriers orthogonal to each other.
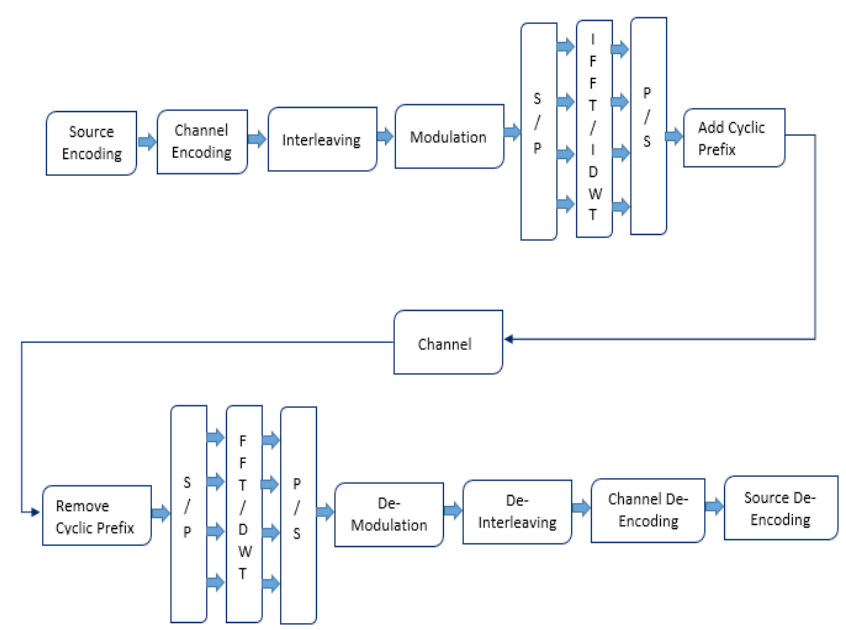

Figure 1. Block diagram of OFDM.

For the case of OFDM transceiver system as shown in Figure 1, a serial to parallel converter is present at the first step which groups the input bit stream which is available from the source encoder in a form of the $\log _{2}$ $\mathrm{M}$ bits, where the $\mathrm{M}$ defines the size of the modulation scheme being used for the modulation process on each of the sub-carriers. This complete process is known as the $\mathrm{S} / \mathrm{P}$ conversion. During this symbol mapping, a sum of $\mathrm{N}$ such symbols is formed which are collectively represented by $\bar{\Xi}^{\prime} X_{m}^{\prime}$. The use of the IFFT helps in the mapping of the sum of the $\mathrm{N}$ symbols to the bins. The formation of the bins from the symbols by the use of IFFT helps in relating the carriers of the specific symbol to be orthogonal to each of the carrier. At last, the demonstrated OFDM symbol is shown as:

$$
x(n)=\frac{1}{N \sum_{m=0}^{N-1}\left(X_{m} e^{\frac{j 2 \pi m n}{N}}\right)} ; 0 \leq n \leq N-1
$$

Here, $X_{m}$ denotes the base-band data which is made available on each one of the defined sub-carrier. Further, the digital to the analog converter creates a process in which the signal which is defined in the time-domain is assumed to be an analog in its nature and is further taken to be passed from the AWGN channel. The relative signal for each of the carrier is taken back for the down 
conversion in the particular form of the discrete N-point sequence which is represented by the $y(n)$. At the reception part, a demodulation process is initiated by the signal which is received and further this process is forwarded by making the usage of the $\mathrm{N}$-point sequence FFT operation on the symbols. The symbol stream which is demodulated expressed in the form:

$y(m)=\sum_{n=0}^{N-1} y(n) e^{-\frac{j 2 \pi m n}{N}}+W(n) ; 0 \leq m \leq N-1$

The Fast Fourier Transform (FFT) for the group of the $\mathrm{w}(\mathrm{n})$ samples is represented by the $\mathrm{W}(\mathrm{m})$, which is mainly assumed to be the Gaussian Noise, which is added in the AWGN channel.

\section{Orthogonality of OFDM Signal}

For the transmission process in the OFDM system, the orthogonal sub-carriers are chosen, that helps in reducing the cross-talk among the sub-channels that eliminate the necessity of the inter-carrier bands. So, this condition helps greatly in simplifying the design pattern of the system for all the sub-channels, which doesn't need any separate filter. The Orthogonality condition necessitates that the spacing in the sub-carrier should be $\Delta \mathrm{f}=k\left(T_{s}\right.$ ) Hertz, where the symbol duration which must be the receiver window size is in seconds and a positive integer $\mathrm{k} \approx 1$. So, for the $\mathrm{N}$ number of sub-carriers, the entire pass-band bandwidth would be defined as $\mathrm{B} \approx \mathrm{N} \Delta \mathrm{f}$ Hertz. Orthogonality has an advantage of improved spectral efficiency as the entire symbol rate corresponds to the Nyquist rate. OFDM demands very precise synchronization in frequency at transmitter and the receiver part. In the existence of deviation in frequency, the relative sub-carriers can never be orthogonal to each other which causes ICI leading to a cross-talk in-between the sub-carriers. The frequency offset occurs mainly due to the mismatch in the sender and the receiver frequencies, which could also be due to the Doppler shift which occurs due to the motion of sender and receiver part. The proper synchronization at both the transmitter and receiver side help in neutralizing the effect of the Doppler Shift. But the effect of such synchronizing action that is done becomes worsen when comes in contact with the multipath fading effects. The effect of this condition arises as a rise in the speed is seen, that in result worsens the situation. So, to conquer this downside, an estimation method was proposed named
Extended Kalman Filter (EKF) in a way for reducing the effect of the ICI on the carriers ${ }^{5}$. This technique is helpful in estimating the normalized values of the CFO optimally in dynamically defined systems ${ }^{6}$. The main motive for a non-linear system was to use the recursive method for the relationship between two variables and also to reduce the error covariance ${ }^{6}$. The expectation of the result which is obtained is the normalized CFO estimated value, which is seen to be close to the observed value so that the offset is minimized in the system. When the integration of the product of two periodic signals over one period equals to 0 , then these two signals shows Orthogonality towards each other.

- For a signal defined in continuous time:

$$
\int_{0}^{T} \operatorname{Cos}\left(2 \pi m f_{0} t\right) \operatorname{Cos}\left(2 \pi n f_{0} t\right) d t=0
$$

- For a signal defined in discrete time:

$$
\sum_{k=0}^{N-1} \operatorname{Cos}\left(\frac{2 \pi k n}{N}\right) \operatorname{Cos}\left(\frac{2 \pi k m}{N}\right) d t=0
$$

In this case, the carriers which are shown to orthogonal to each other do not cause any interference to each other, when they are added together as shown in Figure 2. In the OFDM system, the interference is from the neighboring carriers which do not have frequencies that chase the above relationship. To protect the signals from adjacent channel interference, signals in the system were moved further apart. Each divided sub carrier is placed at a distance allowing it for a $10 \%$ of the guard band. The frequencies on which sub carriers are divided will not be orthogonal to each other but in the case of FDM, we don't bother about the sub-carriers being not orthogonal to each other because the guard band helps in keeping the interference from the carriers under control.

For the practical case, the implementation of the OFDM system is done by the use of DFT technique. During the application of the DFT technique, a basis set is made which is an orthogonal set, in which the signals in the vector space of the DFT forms a linear combination of the orthogonal which are also considered to be orthogonal to each other ${ }^{8}$. One definition of the DFT is considered as the transformation of the input signal that helps in correlating the input signal in respect to each one of the sinusoidal basis functions which are already defined. The 
transformation type is defined by DFT, which is to be used at the transmitter part for the mapping of the input signal onto the predefined set of orthogonally placed subcarriers which are also the orthogonal basis functions. In the same way, the transformation of the signal which is received at the receiver is done for the further processing of the sub-carriers at the receiver side ${ }^{2}$. An absolute estimation of the source signal which received from the sender is done by collecting the signals which are derived from all of the sub carriers. The uncorrelated basic functions formed in the DFT helps in noticing the energy of a sub-carrier for that particularly defined equivalent carrier only. The un-correlation property of the sub-carriers provides an energy which makes no interference between them ${ }^{10}$. Due to this un-correlation only, the overlapping in the spectrum of the sub-carriers occurs without any possibility of any interference between them.

\section{Carrier Frequency Offset (CFO)}

OFDM is a very trendy technique used for the wireless systems that use high data rates for transmission process. This technique is supposed to be very sensitive to the offset that occurs in the frequency and to the synchronization in time. Also, its reactivity to the CFO which is a difference of frequencies at both the transmitter and the receiver side is introducing an ICI in the signal therefore affecting the system's performance. The modulation of the carrier with the signal with the equivalent carrier frequency at the receiver side helps in the conversion of the baseband signal to pass band and then back conversion to baseband level. There are two major issues that are involved with the carrier signal which is used for modulation. One is the phase offset that occurs due to the instability of the carrier signal generators which are used in both the sections. And the other one is the CFO that occurs due to a shift in the Doppler, basically a frequency mismatch between the local oscillators present at the sections. This effect grows to destroy the orthogonality among the sub-carriers. So, its synchronization is necessary for the system. The normalized CFO is divided into two parts, as the integral CFO (IFO) denoted by $\xi_{i}$ and the second is the fractional CFO (FFO) denoted by $\xi_{f}$. Integral one produces a cyclic shift by a factor of $\xi_{i}$ at the receptor section about each sub carrier and in response it doesn't affect the orthogonality between the carrier frequency components whereas the FFO leads to destroying the orthogonality among the sub carriers. The following points define the estimation methods in both the time and the frequency domains as:

- In time domain: There are two techniques that are defined in a time domain. One is the Cyclic Prefix (CP), and the other one is the training sequence. In the $\mathrm{CP}$ method, the estimation of the signal is done by presuming a minute effect of the channel. The CFO from the signal can be obtained from the phase offset present between the $\mathrm{CP}$ and present in its consequent part of the symbol, whereas in the training based method a training sequence is supposed to add up to the front of the OFDM signal for facilitating the estimation of normalized CFO.

- In frequency domain: The techniques defined in the frequency domain mainly includes a comparative analysis of the phase of each of the sub-carrier to its relative successive symbol, and the phase shift obtained in the symbol after this comparison occurs because of the CFO only. The two different modes are defined for the estimation of the CFO in this domain for the pilot method. One is the acquisition mode, and the other one is the tracking. In the case of the first mode i.e. acquisition mode, the estimation of the $\mathrm{CFO}$ occurs on a larger defined range of $\mathrm{CFO}$ and in the other tracking mode, only the estimation of fine $\mathrm{CFO}$ is done.

\subsection{CFO Influence on the OFDM System}

As already mentioned in the above, the major reasons that cause the CFO to rise in the wireless systems are the Doppler frequency shift denoted by $f_{D}$ and the frequency mismatch denoted by $f_{m}$ between the transmitter part and the receiver ${ }^{4}$. If the normalized CFO denoted by $\varepsilon$ is defined as a ratio of a sum $f_{D}+f_{m}$ to the spacing in the sub-carrier denoted by $\mathrm{D} f$, and the OFDM signal received denoted by $r(n)$ for the transmitted signal denoted by s(n) is shown as:

$r(n)=e^{j \frac{2 \pi N\left(f_{D}+f_{m}\right)}{B_{s}}} s(n)=e^{\frac{j 2 \pi n \varepsilon}{N_{F F T}}} s(n)$

Where, $B_{s}$ is defined as the bandwidth of the signal in OFDM and $B_{S}=N_{F F T} \mathrm{D} f$. So, the modulated signal denoted by $R(k)$ can be represented as: 


$$
R_{\varepsilon}(k)=F F T\left[e^{\frac{j 2 \pi n \varepsilon}{N_{F F T}}} s(n)\right]=S(k-\varepsilon)
$$

\section{Methods for Estimating CFO}

CFO is caused due to comparative movement of the transmitter and reception section which causes loss of orthogonality in the sub-carriers resulting in performance degradation and less bandwidth efficiency. Due to its degradation effects, it requires a proper estimation and further its correction too. There are many techniques which can be used for CFO estimation like CP based, Preamble based, Pilot based and Extended Kalman Filter which are discussed in detail below:

\subsection{CP based}

With the use of perfect frame synchronization normalized CFO $(\varepsilon)$ results in $2 \pi n \varepsilon / N F F T$ rotation in the phase of the received symbols $r(n)$. If the channel response is ignored, the phase offset among $\mathrm{CP}$ and its equivalent tail portion of the same symbol caused by $\varepsilon$ was $2 \pi \varepsilon$, since the distance between them is $\mathrm{n}=\mathrm{NFFT}^{3}$. Therefore, the estimated normalized $\mathrm{CFO} \varepsilon$ can be calculated from the offset in terms of phase among $\mathrm{CP}$ and the equivalent tail portion of the same symbol:

$$
\varepsilon=\frac{1}{2 \pi} \arg \left[\sum_{n=1}^{N_{C P}} r^{*}\left(n-N_{C P}+N_{F F T}\right) C(n)\right]
$$

Where, the CP of an OFDM symbol denoted by $C(n)$ and the argument of a complex signal is denoted by $\arg []$. Since the allowed values range for $\arg []$ is $[-\pi, \pi)$, then the estimated normalized CFO range is $[-0.5,0.5)$. But the results can be easily adopted for a common normalization interval, $[0,1)$. This algorithm is called the $\mathrm{CP}$ algorithm.

\subsection{Preamble based}

Preambles based algorithm is moose algorithm which expects adding preambles in the information symbols. In the Moose algorithm, two consequent preambles with the length of NFFT are added in front of the OFDM information symbols. The received symbols with normalized $\mathrm{CFO} \varepsilon$ corresponding to these two preambles $p_{1}(\mathrm{n})$ and $p_{\mathbf{2}}(\mathrm{n})$ have the following relationship:

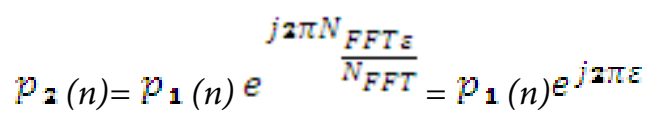

If $P_{\mathbf{2}}(k)$ and $P_{\mathbf{1}}(k)$ are the FFT images of $p_{1}(\mathrm{n})$ and $p_{\mathbf{z}}(\mathrm{n})$, respectively, and considering $\varepsilon$ is a constant the relation between $P_{\mathbf{2}}(k)$ and $P_{\mathbf{1}}(k)$ is as follows:

$$
P_{\mathbf{2}}(k)=F F T\left[P_{\mathbf{2}}(n)\right]=F F T\left[P_{1}(n)^{e^{j \mathbf{2} \pi \varepsilon}}\right]=P_{1}(k) e^{j \mathbf{2} \pi \varepsilon}
$$

Then the normalized $\mathrm{CFO} \varepsilon$ can be estimated using the following equation known as the Moose estimation ${ }^{3}$ algorithm.

$$
\begin{aligned}
& \varepsilon=1 / 2 \pi \arg \left[\sum^{\Sigma}(k=0)^{\dagger}\left(N^{\uparrow}(F F T-1)\right) \ldots\left[P_{\downarrow} 2(k) P_{\downarrow} 1^{\dagger} *(k)=\right]\right. \\
& \varepsilon=1 / 2 \pi \arg \left[\sum_{k=0}^{N F F T-1} p_{\mathbf{2}}(k) p_{\mathbf{1}}(k)\right.
\end{aligned}
$$

The Moose algorithm is very simple and easy to realize, but the estimation precision cannot be guaranteed, especially when SNR is relatively low.

\subsection{Pilot based}

Pilot tones, used in the Classen algorithm ${ }^{\underline{3}}$ to estimate normalized CFO, are inserted in $S(k)$ with fixed distance $D$ and transmitted in every OFDM symbol:

$$
S_{p}(k)=\left\{\begin{array}{cl}
S(k) e^{\frac{j k^{2}}{D N_{F F T}},} & k=0, D, 2 D, \ldots,\left(N_{p}-1\right) D, \\
S(k), & k=\text { other samples },
\end{array}\right\}
$$

Where $\mathrm{Np}=\mathrm{NFFT} / D$ is the number of the pilot tones. Pilot tones can be extracted using FFT transform. The distance between $R 2(k)$ and $R 1(k)$ is NOFDM, where the ratio of the sum $f_{D}+f_{m}$ to subcarrier spacing $\mathrm{D} f$ expresses the normalized $\mathrm{CFO}$ as so the estimation result should be multiplied by a factor NFFT/NOFDM.

Classen's normalized $\mathrm{CFO}$ estimation algorithm can be written as follows:

$$
\begin{gathered}
\frac{\hat{\varepsilon}=\frac{1}{2 \pi} N_{F F T}}{N_{O F D M}\left\{\sum_{m=0}^{N_{p-1}} \frac{\left[\frac{\left[R_{2}\lceil(m D)\right.}{S_{2, p}(m D)}\right]}{\left[\frac{R_{1}(m D)}{S_{1, p}(m D)}\right]}\right\}} \\
=\frac{\frac{1}{2 \pi} N_{F F T}}{N_{O F D M}}\left\{\sum_{m=0}^{N_{p}-1}\left[R_{\mathbf{2}}(m D) S_{2, p}^{*}(m D) S_{1, p}(m D) R_{\mathbf{i}}^{*}(m D)\right]\right\}
\end{gathered}
$$

Where, the subscripts " 1 " and " 2 " denote first or second OFDM symbol with pilot tones. The Classen algorithm makes use of the quotient between $R(k)$ and $S(k)$ to accurately estimate the value of normalized CFO. The estimation result should be more accurate than in the case 
of $\mathrm{CP}$ and the Moose algorithm ${ }^{4}$. However, adding pilot tones adds extra work for the transmitter, increases the estimation algorithm's complexity, and requires dedication of at least two OFDM symbols that cannot transmit information.

\subsection{Extended Kalman Filter}

The filter that shows linearization about the current mean and covariance is referred to as an Extended Kalman Filter (EKF). In the OFDM systems, the EKF has been engaged in estimating and tracking the offsets caused due to the symbol timing and the frequency in the time domain ${ }^{6,7}$. Still, this type of method works reliable in an environment where the presumptions are made on the Gaussianity of the noises and the state hold. An EKF ${ }^{4}$ was applied to determine the normalized offset in frequency with the usage of the training sequence technique which was already known at the transmitter and reception section. The training sequence taken was the preamble having 256 symbols in it. The training sequence based implemented the method which is used was computationally simpler and effortlessly employed on the real time practical systems. The filter has mainly two-steps i.e. the prediction step and the update step. In the first prediction step, in which the next future state of the system was being predicted provided the previous measurements and in the second updation step, in which the current state of the system was being estimated given the measurement at that time step. The received OFDM signal $y(n)$ can be expressed as:

$y(n)=x(n) \exp (j 2 \pi n \varepsilon(n) / N)+w(n)$

The expression is given $\exp (j 2 \pi n \varepsilon(n) / \mathrm{N})$ shows the shift in frequency $(\varepsilon)$ was considered as the offset in normalized frequency and shown as $\Delta \mathrm{fNTs}$, in which $\Delta \mathrm{f}$ gives the difference in frequency among the $\mathrm{Tx}$ and $\mathrm{Rx}$ carrier frequencies. Where $w(n)$ was the AWGN included in the channel and the $y(n)$ is having a linear relationship with the desired value of $\varepsilon(n)$. The ICI distortion due to CFO in the data symbols which are denoted by $\mathrm{x}(\mathrm{n})$ follows a training sequence could be improved by applying the multiplication on the data symbols represented by $y(n)$, which are obtained from a complex conjugate of the frequency offset which is estimated and by further applying the FFT forms as follows:

$$
x^{\mathbf{I}}(n)=F F T\{y(n) \exp (-j 2 \pi n \varepsilon / N)\}
$$

In the above presented estimated expression using the EKF technique, it is was observed that the channel which is being used is varying slowly with respect to time so that during the transmission of one OFDM frame, in order to maintain a approximate balance of the time-variant channel impulse response to the quasi-static and also in those situations in which the symbols were transmitted simultaneously with an earlier set of the preambles ${ }^{4}$. So, the offset in frequency was taken to be a constant value all through the period of a frame which allows the application of the preamble as a training sequence for further estimation. All the required results were achieved by the transmission of the signals via AWGN channel.

\section{Comparison Analysis of Various Methods}

CFO estimation is done by using four different techniques. First one was by the usage of the phase difference calculated between the $\mathrm{CP}$ and the equivalent real part of an OFDM symbol. The second was by the phase difference which calculated between the two repetitive preambles. The third one was by considering the estimation between pilot tones in the two consecutive OFDM symbols. MSE performance for CFO estimation using these three different techniques is evaluated, and simulation results conclude that Pilot tone method is superior to the $\mathrm{CP}$ and the Preamble based method. The performance base of these estimation techniques also varies depending upon the sum of the samples being considered in CP and preamble and the number of pilot tones being used for CFO estimation. As pilot method better of all the above three methods, now we are comparing this method with Kalman Filter. The research keeps its focus on the improvement in performance of the system in the presence of the frequency offset. The estimation was done by the use of Kalman Filters without any reduction of the bandwidth efficiency still, it will require a complex implementation at the $\mathrm{Rx}$ section which necessitates a high processing speed. The model is taken forward with the presumption of keeping the AWGN channel for transmission. The suggested algorithm is considered to be independent of the CSI as it uses the null sub-carriers and also it does not need any training sequence. Simulations conclude that the proposed method is supposed to be providing the robustness against the noise as well as the Doppler shifts. As it is capable of tracking the CFO (accurately) whenever the frame is changing through the transmission. 


\section{Conclusion}

The CFO effect is seen mainly as a major downside for the OFDM based systems, which leads to a loss in Orthogonality of sub-carriers resulting in inter carrier interference in the symbols due to which there is performance degradation that occurs in the system which disturbs its robustness for multipath fading and also affecting the efficiency in the spectra's. So, a proper estimation of this effect and its compensation is necessary for the system to work more efficiently. The above-presented techniques are helpful in mitigating the CFO effect from the system. In this research, a comparative analysis of the four methods is done in order to find the most resourceful one of all the techniques and the performance analysis shown by them reveals that the EKF method was very helpful in the estimation and tracking of CFO effect more effectively in the system and that helps in providing good performance in the system in the presence of frequency offset and provides more accuracy in the OFDM system.

\section{References}

1. Weinstein SB, Ebert PM. Data transmission by frequencydivision multiplexing using the discrete fourier transform. IEEE Transactions on Communications. 1971; 19(5):62834.

2. Li YG, Stuber GL. OFDM for Wireless Communications. Springer Publication; 2006.
3. Nishad PK, Singh P. Carrier frequency offset estimation in OFDM systems. IEEE Conference on Information and Communication Technology; Jeju Island. 2013. p. 885-9.

4. Senevirathna SB, Jayawardena C, Perera SS, Perera CL, Ranasignhe D, Wijerathna SR, Bandara TN. Carrier frequency offset estimation for OFDM system using extended Kalman Filter. IEEE Conference on Information and Automation for Sustainability; Colombo. 2008. p. 351-4.

5. Haykin S. Kalman Filtering and Neural Networks. John Wiley and Sons; 2001.

6. Chen R, Wang X, Liu JS. Adaptive joint detection and decoding in flat-fading channels via mixture Kalman filtering. IEEE Transaction Information Theory. 2000; 46(6):2079-94.

7. Roman T, Enescu M, Koivunen Y. Joint time-domain tracking of channel and frequency offset for OFDM systems. IEEE Workshop on Signal Processing Advances in Wireless Communications, SPAWC; Rome, Italy. 2003. p. 605-9.

8. Kalaivani D, Karthikeyen S. VLSI implementation of areaefficient and low power OFDM transmitter and receiver. Indian Journal of Science and Technology. 2015 Aug; 8(18). Doi no:10.17485/ijst/2015/v8i18/63062

9. Avila J, Thenmozhi K. Let multiband-OFDM modified by wavelet. Indian Journal of Science and Technology. 2014 Jan; 7(8). Doi no: 10.17485/ijst/2014/v7i8/42090

10. Vadivel $M$. Optimization of radio resource allocation in energy efficient OFDMA systems. Indian Journal of Science and Technology. 2015 Feb; 8(S3). Doi no: 10.17485/ ijst/2015/v8iS3/62296 